\title{
Design and Implementation of the Inference Machine of an Intelligent Ultrasonic Emuls ification Instrument
}

\author{
Aili Li, \\ School of computer \& communication \\ University of Science \& Technology Beijing \\ Beijing, China \\ e-mail:1laall88@sina.com \\ Zhiwen Yang, \\ School of computer \& communic ation \\ University of Science \& Technology Beijing \\ Beijing, China \\ e-mail: 1sj28219@163.com
}

\begin{abstract}
Based on the research of the intelligent cataract ultrasonic emulsification instrument, mainly discusses the principle of design and inference algorithm of the inference machine of intelligent ultrasonic emulsification instrument. Designing the cataract expert knowledge base by the use of relational database, the inference machine can simulate the real doctor thinking features effectively; at the same time, according to the patient's cataract symptoms, it can make intelligent judgment to select the ultrasonic energy level for the emulsifying needle, and effectively control the release of energy. The inference strategy is the combined of deductive reasoning, certainty and forward inference, so as to improve the efficiency of inference machine. Through the verification tests, the system obtains better inference results.
\end{abstract}

Keywords-Intelligent; Cataract; Relational Database; Inference Machine; Inference Strategy;

\section{INTRODUCTION}

In China, there is a large number of cataract diseases, the main treatment for the disease is surgical removal. But the ultrasonic emulsification instrument suction technology currently used are still very low degree of automation, overreliance on operation physician specialist technical level and other defects. So it is necessary to study the method of identification cataract hardness according to the operation video image, provide a basis for energy release automation control according to the hardness.

Studies have shown that in the process of cataract ultrasonic emulsification operation, ultrasonic energy release is the key to the success of cataract operation[1].Accurately identify the hardness of the nucleus of the lens, effectively control the release of the ultrasonic energy, so that the energy emitted by the probe just to achieve emulsification and aspiration of the lens nucleus block, without excess energy wound normal tissue, or insufficient energy make the emulsified not sufficient.

The design of inference machine plays an important role in the recognition system of ultrasonic emulsification instrument. The core of the inference machine is to control

\author{
Xuyan $\mathrm{Tu}$, \\ School of computer \& communication \\ University of Science \& Technology Beijing \\ Beijing, China \\ Xuefeng Zheng, \\ School of computer \& communication \\ University of Science \& Technology Beijing \\ Beijing, China
}

and coordinate the entire recognition system; The reasoning is reasoning and logical thinking process simulation medical experts, the use of the knowledge base has been formed to derive a reasonable conclusion; Reasoning mechanism by which reasoning strategies to achieve reasoning functions [2].In this paper, based on the division of a large number of cataract patients nuclear hardness classification, build expert knowledge base to complete the design and implementation of the ultrasonic emulsification instrument inference machine.

\section{REQUIREMENT S FOR THE DESIGN OF INFERENCE MACHINE}

In this paper, before the ultrasonic emulsification instrument inference machine design, focus on the specific require ments of the inference machine design.

Related studies show that, effects of the inference engine reasoning and reasoning efficiency is the key parts of the measure of the inference machine design ${ }^{[3]}$.

Good and bad effects of inference has a great relationship with the application of knowledge of thinking, generally have the following four basic requirements: (1) Valid information can be selected according to the accuracy of their environment; (2) Accurate use of the information which has been selected; (3) By the new way or path further inference; (4) The end of the inference process at the appropriate time.

The reasoning efficiency is mainly related to the following two factors: First, select effective information required for consideration; Secondly, the way of use this effective information.

Design an efficient inference machine, issues to consider is how to make more balanced between inference costs and control costs, or the introduction of new technology to control the reasoning strategy to improve the efficiency of the inference machine reasoning ${ }^{[4]}$. 


\section{IMPLEMENTATION OFTHE INFERENCE CONTROL STRATEGY}

\section{A. Inference Mechanism}

An inference comprehensive and efficient inference mechanism is the key to intelligent expert knowledge systems research, good inference mechanism is very important for the inference machine. Medical experts' accumulated experience in long-term cataract surgery is abstract and general knowledge. But in the actual operation according to the image color, texture and other characteristics to determine the lens nucleus hardness level, and then the energy specific adjustment probe emulsification instrument release, is an adjustment process from a general to specific ${ }^{[5]}$.

At present, the inference machine drive mode mainly has forward inference (i.e. forward reasoning), backward inference (i.e. the reverse inference) and two-way inference in three ways ${ }^{[6]}$. Considering the tight inference ultrasonic emulsification instrument recognition system, the method of the method of strict control should be similar to human medical experts to solve problems ${ }^{[7]}$; this paper adopts fuzzy inference and forward inference (Forward Reasoning) reas oning mechanism combining.

\section{B. Fuzzy Inference Mechanism}

Fuzzy inference mechanism is based on the fuzzy control rules by the premise to infer conclusions. During the research, the fuzzy expert, taking into account the system controller controls the decision depends mainly on the characteristic of the input data. Therefore, we use data-driven forward inference strategy. According to the actual input signal, compared with the control rules in the rule database, if it meets the rule then execute and start output, otherwise continue to traverse the search until the traverse to the target so far.

In the actual control process, the computer make periodic sampling for the two input signals of the nuclear hardness and the size of the cataract, make fuzzy process and calculating the membership value, and then follow the above rule to do reasoning. The method "min-max" reasoning is commonly used, minimum membership of prerequisite rules are the satisfaction of the conclusions of the rule-based reasoning, finally, the maximum value of the rules is the result of the reasoning, expressed by the formula as follows:

$$
\begin{gathered}
\mu(n)=\operatorname{MIN}\left\{\mu(\mathrm{YD})^{(\mathrm{n})}, \mu(\mathrm{TJ})^{(\mathrm{n})}\right\} \quad n=1,2, \cdots N \\
\mu(N L)=\operatorname{MAX}_{n=1}^{N} \mu(n)
\end{gathered}
$$

Where, $\mathrm{N}$ is the total number of fuzzy control ru les in the control rules database.

\section{THE REALIZAT ION OF ULTRASONIC EMULSIFICATION INST RUMENT INFERENCE MACHINE}

\section{A. The Establishment of Fuzzy Expert Knowledge}

Fuzzy expert knowledge base includes fuzzy database and control rules database ${ }^{[8]}$.

\section{1) Fuzzy Database}

Fuzzy database stores various parameters associated with fuzzy data processing, in detail, including a variety of language data quantization factor, scale factor, fuzzy space segmentation and membership function and so on ${ }^{[9]}$. The following describes the scale transformation factor.

The input data is multiplied by the corresponding quantization factor to complete the input data conversion from the basic to the fuzzy domain; the precise control (ultrasonic energy amount) multiplied by the corresponding scale factor, can convert from fuzzy domain to the corresponding basic domain, in order to control the object ${ }^{[10]}$.

The range of the design of the precise variables are not symmetrical distribution in $[-\mathrm{m}, \mathrm{m}]$, but in $[0, \mathrm{~m}]$, which makes the scale conversion factor becomes more simple. Such as $\mathrm{x} \in[0, \mathrm{a}]$ is converted to $\mathrm{x} \mathrm{x}^{\prime} \in[0, \mathrm{~b}]$, then the scale conversion factor is

$$
x^{\prime}=\frac{b}{a} x
$$

\section{2) The Control Rules Database}

The control rules database stores a certain number of fuzzy control rules, these rules include fuzzy experience and knowledge of cataract ultrasonic emulsification operation experts and control technical personnel, these rules are called by the inference machine, and then complete control of ultras onic emulsification energy.

Fuzzy control rules are created based on medical diagnostic knowledge. In order to establish these rules, we need to solve the problem of accessing diagnostic information. At present, the way to acquire knowledge mainly have the following several kinds: (1) artificial obtain, directly acquire knowledge from domain experts, after the processing and handling, transfer into the knowledge base by programming; (2) semi-automatic acquisition, the use of specific knowledge compiler, medical experts transfer relevant diagnostic information into the content of the knowledge base; (3) the automatic knowledge acquisition, which according to the feedback information of the closedloop control system, through its learning system directly acquire knowledge from the control process and store in knowledge base, constantly revised and refined the content of the knowledge base. The design for specific situations and use the first kind of knowledge acquisition method, gather knowledge and experience of clinical operations data from cataract experts and access a lot of relevant information, after analysis, classified and summarized, use appropriate language to describe, and ultimately extract a complete set of fuzzy control rules.

Fuzzy control strategy that is adopted in this paper is constituted by "IF-THEN" type of statement, data variables of these statements is the cataract lens nucleus hardness level, the output conclusion is the energy release amount of ultrasonic emulsification instrument probe. In actual use, usually express the fuzzy control strategy in the form of the two-dimensional table, as shown in Table 1.

Two input variables the hardness level YD and size of TJ respectively with five and nine fuzzy linguistic variables; it can generate 45 control rules. Use usual left-to-right, top- 
down numbering sequence, the control rules is explained as follows:

Rule 1: if $\mathrm{YD}$ is $\mathrm{Y} 1$ and $\mathrm{TJ}$ is $\mathrm{T} 1$ then $\mathrm{NL}$ is $\mathrm{N} 1$

TABLE I. CONTROL RULES TABLE.

\begin{tabular}{|c|c|c|c|c|c|}
\hline TJ & Y1 & $\mathrm{Y} 2$ & $\mathrm{Y} 3$ & $\mathrm{Y} 4$ & $\mathrm{Y} 5$ \\
\hline $\mathrm{T} 1$ & & & & & \\
\hline $\mathrm{T} 2$ & $\mathrm{~N} 1$ & $\mathrm{~N} 1$ & $\mathrm{~N} 2$ & $\mathrm{~N} 2$ & $\mathrm{~N} 3$ \\
\hline $\mathrm{T} 3$ & $\mathrm{~N} 1$ & $\mathrm{~N} 2$ & $\mathrm{~N} 3$ & $\mathrm{~N} 3$ & $\mathrm{~N} 4$ \\
\hline $\mathrm{T} 4$ & $\mathrm{~N} 1$ & $\mathrm{~N} 3$ & $\mathrm{~N} 3$ & $\mathrm{~N} 4$ & $\mathrm{~N} 4$ \\
\hline $\mathrm{T} 5$ & $\mathrm{~N} 2$ & $\mathrm{~N} 3$ & $\mathrm{~N} 4$ & $\mathrm{~N} 4$ & $\mathrm{~N} 5$ \\
\hline $\mathrm{T} 6$ & $\mathrm{~N} 2$ & $\mathrm{~N} 3$ & $\mathrm{~N} 4$ & $\mathrm{~N} 4$ & $\mathrm{~N} 5$ \\
\hline $\mathrm{T} 7$ & $\mathrm{~N} 2$ & $\mathrm{~N} 3$ & $\mathrm{~N} 4$ & $\mathrm{~N} 5$ & $\mathrm{~N} 5$ \\
\hline $\mathrm{T} 8$ & $\mathrm{~N} 2$ & $\mathrm{~N} 3$ & $\mathrm{~N} 5$ & $\mathrm{~N} 5$ & $\mathrm{~N} 6$ \\
\hline $\mathrm{T} 9$ & $\mathrm{~N} 2$ & $\mathrm{~N} 4$ & $\mathrm{~N} 5$ & $\mathrm{~N} 5$ & $\mathrm{~N} 6$ \\
\hline
\end{tabular}

Rule 2: if $\mathrm{YD}$ is $\mathrm{Y} 2$ and $\mathrm{TJ}$ is $\mathrm{T} 1$ then $\mathrm{NL}$ is $\mathrm{N} 1$

Rule 3: if $\mathrm{YD}$ is $\mathrm{Y} 3$ and $\mathrm{TJ}$ is $\mathrm{T} 1$ then $\mathrm{NL}$ is $\mathrm{N} 2$ $\cdots$

Rule 44: if YD is Y4 and TJ is T9 then NL is N5 Rule 45: if YD is $\mathrm{Y} 5$ and $\mathrm{TJ}$ is $\mathrm{T} 9$ then NL is N6

In the experiment, no matter what method, the control rules are approximate, and the above-described control rule is no exception. We must correctly solve the problem of the coordination of these rules. In our study, not only to ensure the completeness of the rules, that is, for any input, there must be a reasonable output, but also to ensure the mutual compatibility of the rules, that any two rules cannot get conflicting conclusions.

\section{EXPERIMENTAL RESULTS}

In accordance with the classification criteria of the cataract hardness level, the establishment of a training library and test library. All images are taken from the real-time ultrasonic emulsification video of Beijing Tongren Hos pital Eye Center.

The image library is divided into training library and test library. Training library contains 649 pictures, test library contains 647 pictures, and images are shown in Figure 1. Table 2 shows the details of the training library and test library.

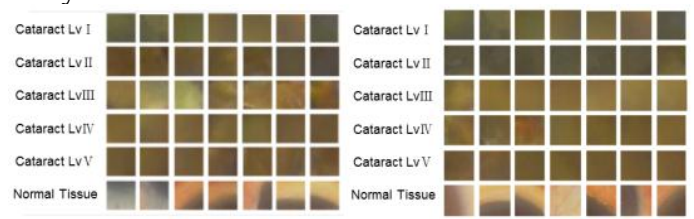

Figure 1. Experimental Image.

TABLE II. LIST OF THE EXPERIMENTAL PICTURE AMOUNT.

\begin{tabular}{|c|c|c|}
\hline Classification & Training library & Test library \\
\hline Cataract I Level & 293 & 296 \\
\hline Cataract II Level & 184 & 183 \\
\hline Cataract III level & 37 & 33 \\
\hline Cataract IV level & 28 & 30 \\
\hline Cataract V level & 12 & 14 \\
\hline Normal tissue & 95 & 91 \\
\hline Total & 649 & 647 \\
\hline
\end{tabular}

When the ultrasonic emulsification instrument identification system startup, to capture video during surgery, the first thing to do is pretreatment on video image acquired, at the same time, detecting the trajectory of the ultrasonic emulsification instrument probe, to realize the dynamic tracking of ultrasonic emulsification instrument probe, then extract the image ahead the probe, carries on the identification; if the result is the normal tissue, the emulsification instrument does not release energy; if it is the cataract, recognize its hardness, according to forward inference mechanis $m$ and the medical experts knowledge base, judge the energy amount should the probe release, then the recognition result is output to the control system which controls the ultrasonic emulsification instrument to release energy, make the emulsion of cataract. The identification process is shown in Figure 2; the experimental result is shown in Figure 3.

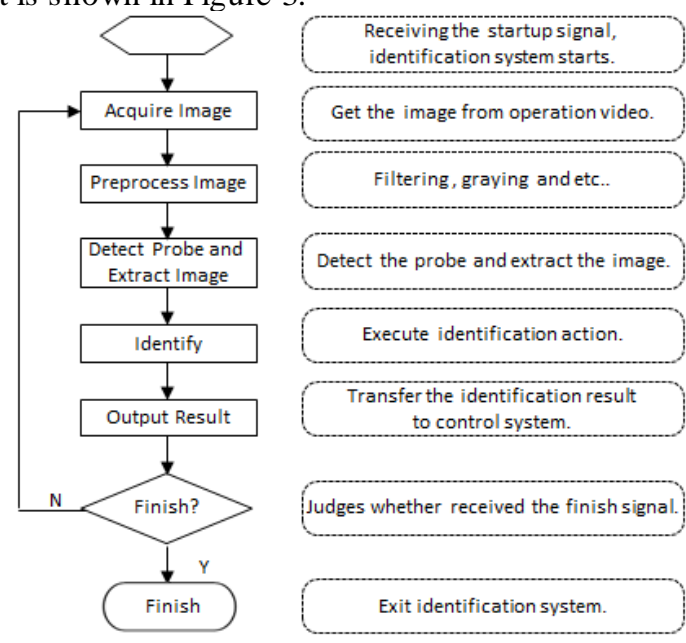

Figure 2. The Flow Chart of Identification System. 


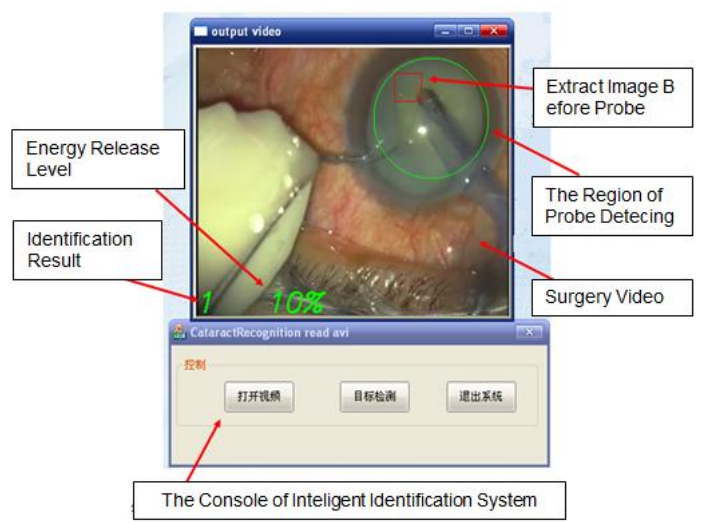

Figure 3. The System Running in Experiments.

\section{CONCLUSIONS}

The inference machine is the thinking structure of ultrasonic emulsification instrument identification system, and important component of the identification system ${ }^{[11]}$. In this paper, the design of the inference machine, preferably simulate the judgment process of medical experts on the hardness of the cataract lens nucleus, and thus provide a reliable bas is for the precise control and execute the energy release for the probe. The ultrasonic emulsification instrument based on the inference engine achieved more satisfactory results in cataract surgery clinical trials.

\section{REFERENCES}

[1] Siquan Zhu, Hongwei Hao, Xisheng Li. National Science and Technology Support Program (Project number 2006BAI03A09) - the efficient intelligent minimally invasive cataract treatment system developed mission statement. 2006.

[2] $\mathrm{Li}$ Gao, Huaiyuan $\mathrm{Pu}$, Shu Hu. Design and Implementation of an intelligent medical diagnostic inference machine [J]. Computer Applications and Soft ware, 2002, (6): 44-46.

[3] Jin Li, Hanxing Lv. Design and implementation of an inference engine of the medical diagnosis expert system [J]. Microcomputer Development.2004 (9):42-44.

[4] Yanqi Luo, Leiting Chen. Method of knowledge representation in expert system [J]. Elect ronic computer, 2001, 8(151): 28-31.

[5] Zidu Zhao. Inference mechanism and reasoning methods [J]. Automation Expo. 1997(06).

[6] Lijun Cao, Xiaofeng Hou,Jian Huang. Application of hybrid inference mechanism in fault forecast system for guns [J]. Journal of Gun Launch \& Control.2005 (3).

[7] Deyun Chen, Xiuzhong Li, Shirong Chen. Expert system tight control and compet ition rules processing method [J]. Computer Engineering and Applications, 1994, 3(4):18- 20.

[8] Fanyou Zhang, Jixin Zou, Song Lin. The application of dual linear ultrasonic equipment for phacoemulsification of hard nucleus cat aract [J]. Chinese Journal of Practical Ophthalmology, 2004, 2(22):114-115.

[9] Juan Li Self-tuning factor fuzzy PID controller design and research [M]. 2007 Jiangsu University: Control Theory and Control Engineering.

[10] Jiangang Peng. Study on fuzzy control of the beam and ball system [J]. Automation Technology and Application. 2008 (3).

[11] Wei Wang, Jianjun He. Design and implementation of an inference engine of the pneumatic drying expert system [J]. Automation \& Instrumentation, 2007(2):15-17. 